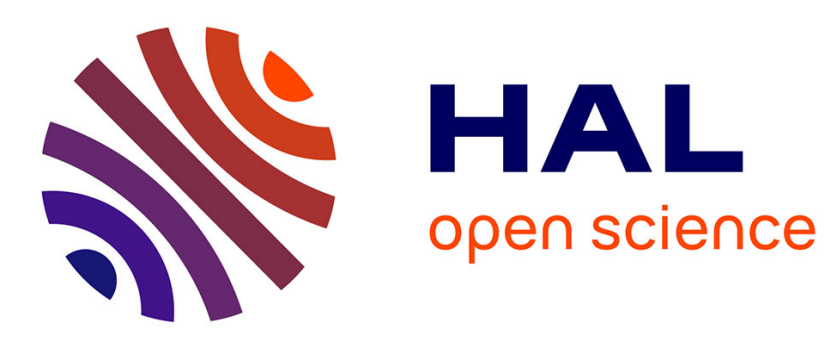

\title{
Fast Tuning of Intra-Cluster Collective Communications
}

Luiz Angelo Barchet-Estefanel, Grégory Mounié

\section{To cite this version:}

Luiz Angelo Barchet-Estefanel, Grégory Mounié. Fast Tuning of Intra-Cluster Collective Communications. EuroPVM/MPI 2004 11th European PVM/MPI Users' Group Meeting, 2004, France. pp.28-35, 10.1007/978-3-540-30218-6_12 . hal-00002548v3

\section{HAL Id: hal-00002548 \\ https://hal.science/hal-00002548v3}

Submitted on 2 Dec 2004

HAL is a multi-disciplinary open access archive for the deposit and dissemination of scientific research documents, whether they are published or not. The documents may come from teaching and research institutions in France or abroad, or from public or private research centers.
L'archive ouverte pluridisciplinaire $\mathbf{H A L}$, est destinée au dépôt et à la diffusion de documents scientifiques de niveau recherche, publiés ou non, émanant des établissements d'enseignement et de recherche français ou étrangers, des laboratoires publics ou privés. 


\title{
Fast Tuning of Intra-Cluster Collective Communications
}

\author{
Luiz Angelo Barchet-Estefanel`, Grégory Mounié \\ Laboratoire ID - IMAG, Project APACHE ${ }^{\star \star}$ \\ 51, Avenue Jean Kuntzmann, F38330 Montbonnot St. Martin, France \\ \{Luiz-Angelo.Estef anel, Gregory. Mounie\}@imag.fr
}

\begin{abstract}
Recent works try to optimise collective communication in grid systems focusing mostly on the optimisation of communications among different clusters. We believe that intra-cluster collective communications should also be optimised, as a way to improve the overall efficiency and to allow the construction of multi-level collective operations. Indeed, inside homogeneous clusters, a simple optimisation approach rely on the comparison from different implementation strategies, through their communication models. In this paper we evaluate this approach, comparing different implementation strategies with their predicted performances. As a result, we are able to choose the communication strategy that better adapts to each network environment.
\end{abstract}

\section{Introduction}

The optimisation of collective communications in grids is a complex task because the inherent heterogeneity of the network forbids the use of general solutions. Indeed, the optimisation cost can be fairly reduced if we consider grids as interconnected islands of homogeneous clusters, if we can identify the network topology.

Most systems only separate inter and intra-cluster communications, optimising communication across wide-area networks, which are usually slower than communication inside LANs. Some examples of this "two-layered" approach include ECO [11], MagPIe [4] and even LAM-MPI 7 [8]. While ECO and MagPIe apply this concept for wide-area networks, LAM-MPI 7 applies it to SMP clusters, where each SMP machine is an island of fast communication. Even though, there is no real restriction on the number of layer and, indeed, the performance of collective communications can still be improved by the use of multi-level communication layers, as observed by [3].

If most works today use the "islands of clusters" approach, to our knowledge none of them tries to optimise the intra-cluster communication. We believe that while inter-cluster communication represents the most important aspect in gridlike environments, intra-cluster optimisation also should be considered, specially

* Supported by grant BEX 1364/00-6 from CAPES - Brazil
** This project is supported by CNRS, INPG, INRIA and UJF 
if the clusters should be structured in multiple layers [3]. In fact, collective communications in local-area networks can still be improved with the use of message segmentation [1, 6] or the use of different communication strategies [12].

In this paper we propose the use of well known techniques for collective communication, that due to the relative homogeneity inside each cluster, may reduce the optimisation cost. Contrarily to [13], we decided to model the performance of different implementation strategies for collective communications and to select, according to the network characteristics, the most adapted implementation technique for each set of parameters (communication pattern, message size, number of processes). Hence, in this paper we illustrate our approach with two examples, the Broadcast and Scatter operations, and we validate our approach by comparing the performance from real communications and the models' predictions.

The rest of this paper is organised as follows: Section 2 presents the definitions and the test environment we will consider along this paper. Section 3 presents the communication models we developed for both Broadcast's and Scatter's most usual implementations. In Section 4 we compare the predictions from the models with experimental results. Finally, Section 5 presents our conclusions, as well as the future directions of the research.

\section{System Model and Definitions}

In this paper we model collective communications using the parameterised $\log P$ model, or simply pLogP [6]. Hence, all along this paper we shall use the same terminology from pLogP's definition, such as $g(m)$ for the gap of a message of size $m, L$ as the communication latency between two nodes, and $P$ as the number of nodes. In the case of message segmentation, the segment size $s$ of the message $m$ is a multiple of the size of the basic datatype to be transmitted, and it splits the initial message $m$ into $k$ segments. Thus, $g(s)$ represents the gap of a segment with size $s$.

The pLogP parameters used to feed our models were previously obtained with the MPI LogP Benchmark tool [5] using LAM-MPI 6.5.9 [7]. The experiments to obtain pLogP parameters, as well as the practical experiments, were conducted on the ID/HP icluster-1 from the ID laboratory Cluster Computing Centre ${ }^{1}$, with 50 Pentium III machines (850Mhz, 256MB) interconnected by a switched Ethernet 100 Mbps network.

\section{Communication Models with pLogP}

Due to the limited space, we cannot present models for all collective communication, thus we chose to present the Broadcast and the Scatter operations. Although they are two of the simplest collective communication patterns, practical implementations of MPI usually construct other collective operations, as for example, Barrier, Reduce and Gather, in a very similar way, what makes these two

\footnotetext{
$\overline{{ }^{1} \text { http://www-id.imag.fr/Grappes/ }}$
} 
operations a good example for our models accuracy. Further, the optimisation of grid-aware collective communications explores intensively such communication patterns, as for example the AllGather operation in MagPIe, which has three steps: a Gather operation inside each cluster, an AllGatherv among the clusters' roots and a Broadcast to the cluster's members.

\subsection{Broadcast}

With Broadcast, a single process, called root, sends the same message of size $m$ to all other $(P-1)$ processes. Among the classical implementations for broadcast in homogeneous environments we can find flat, binary and binomial trees, as well as chains (or pipelines). It is usual to apply different strategies within these techniques according to the message size, as for example, the use of a rendezvous message that prepares the receiver to the incoming of a large message, or the use of non-blocking primitives to improve communication overlap. Based on the models proposed by [6], we developed the communication models for some current techniques and their "flavours", which are presented on Table 1 .

We also considered message segmentation [6, 12], which may improve the communication performance under some specific situations. An important aspect, when dealing with message segmentation, is to determine the optimal segment size. Too little messages pay more for their headers than for their content, while too large messages do not explore enough the network bandwidth. Hence, we can use the communication models presented on Table 1 to search the segment size $s$ that minimises the communication time in a given network. Once determined this segment size $s$, large messages can be split into $\lfloor m / s\rfloor$ segments, while smaller messages will be transmitted without segmentation.

As most of these variations are clearly expensive, we did not consider them on the experiments from Section 4, and focused only in the comparison of the most efficient techniques, the Binomial and the Segmented Chain Broadcasts.

\subsection{Scatter}

The Scatter operation, which is also called "personalised broadcast", is an operation where the root holds $m \times P$ data items that should be equally distributed among the $\mathrm{P}$ processes, including itself. It is believed that optimal algorithms for homogeneous networks use flat trees [6], and by this reason, the Flat Tree approach is the default Scatter implementation in most MPI implementations. The idea behind a Flat Tree Scatter is that, as each node shall receive a different message, the root shall sends these messages directly to each destination node.

To better explore our approach, we constructed the communication model for other strategies (Table 2) and, in this paper, we compare Flat Scatter and Binomial Scatter in real experiments. In a first look, a Binomial Scatter is not as efficient as the Flat Scatter, because each node receives from the parent node its message as well as the set of messages it shall send to its successors. On the other hand, the cost to send these "combined" messages (where most part is useless to the receiver and should be forwarded again) may be compensate 
Table1. Communication Models for Broadcast

\begin{tabular}{|c|c|}
\hline Implementation Technique & Communication Model \\
\hline \hline Flat Tree & $(P-1) \times g(m)+L$ \\
\hline Flat Tree Rendezvous & $(P-1) \times g(m)+2 \times g(1)+3 \times L$ \\
\hline Segmented Flat Tree & $(P-1) \times(g(s) \times k)+L$ \\
\hline Chain & $(P-1) \times(g(m)+L)$ \\
\hline Chain Rendezvous & $(P-1) \times(g(m)+2 \times g(1)+3 \times L)$ \\
\hline Segmented Chain (Pipeline) & $(P-1) \times(g(s)+L)+(g(s) \times(k-1))$ \\
\hline Binary Tree & $\leq\left\lceil\log _{2} P\right\rceil \times(2 \times g(m)+L)$ \\
\hline Binomial Tree & $\left\lfloor\log _{2} P\right\rfloor \times g(m)+\left\lceil\log _{2} P\right\rceil \times L$ \\
\hline Binomial Tree Rendezvous & $\left\lfloor\log _{2} P\right\rfloor \times g(m)+\left\lceil\log _{2} P\right\rceil \times(2 \times g(1)+3 \times L)$ \\
\hline Segmented Binomial Tree & $\left\lfloor\log _{2} P\right\rfloor \times g(s) \times k+\left\lceil\log _{2} P\right\rceil \times L$ \\
\hline
\end{tabular}

by the possibility to execute parallel transmissions. As the trade-off between transmission cost and parallel sends is represented in our models, we can evaluate the advantages of each model according to the clusters' characteristics.

Table2. Communication Models for Scatter

\begin{tabular}{|c|c|}
\hline Implementation Technique & Communication Model \\
\hline \hline Flat Tree & $(P-1) \times g(m)+L$ \\
\hline Chain & $\sum_{j=1}^{P-1} g(j \times m)+(P-1) \times L$ \\
\hline Binomial Tree & $\sum_{j=0}^{\left|\log _{2} P\right|-1} g\left(2^{j} \times m\right)+\left\lceil\log _{2} P\right\rceil \times L$ \\
\hline
\end{tabular}

\section{Practical Results}

\subsection{Broadcast}

To evaluate the accuracy of our optimisation approach, we measured the completion time of the Binomial and the Segmented Chain Broadcasts, and we compared these results with the model predictions. Through the analysis of Figs. 1(a) and 11(b), we can verify that models' predictions follow closely the real experiments. Indeed, both experiments and models predictions show that the Segmented Chain Broadcast is the most adapted strategy to our network parameter, and consequently, we can rely on the models' predictions to chose the strategy we will apply.

Although models were accurate enough to select the best adapted strategy, a close look at the Fig. 1 still shows some differences between model's predictions and the real results. We can observe that, in the case of the Binomial Broadcast, there is a non expected delay when messages are small. In the case of 


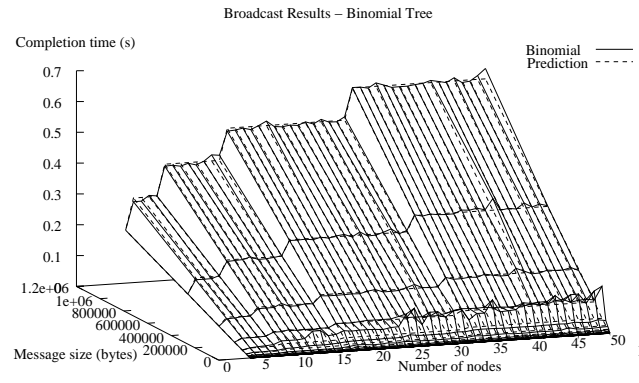

(a) Binomial Tree

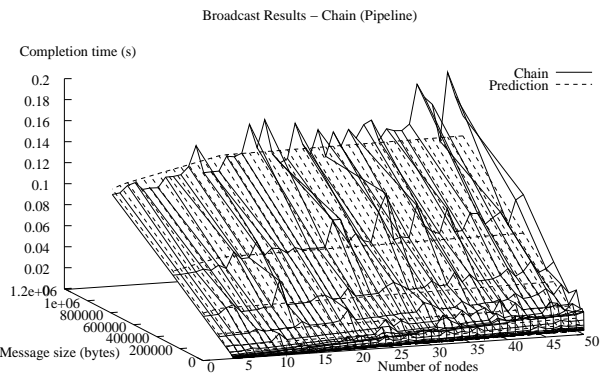

(b) Segmented Chain - 8kB segments

Figure1. Comparison between models and real results

the Segmented Chain Broadcast, however, the execution time is slightly larger than expected. Actually, we believe that both variations derive from the same problem.

Hence, we present in Fig. 2 the comparison of both strategies and their predictions for a fixed number of machines. We can observe that predictions for the Binomial Broadcast fit with enough accuracy the experimental results, except in the case of small messages (less than $128 \mathrm{kB}$ ). Actually, similar discrepancies were already observed by the LAM-MPI team, and according to [9,10, they are due to the TCP acknowledgement policy on Linux that may delay the transmission of some small messages even when the TCP_NODELAY socket option is active (actually, only one every $n$ messages is delayed, with $n$ varying from kernel to kernel implementation).

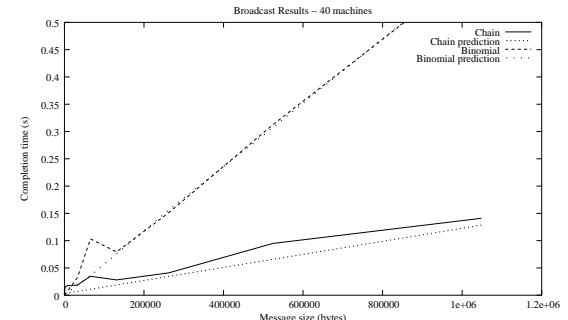

Figure2. Comparison between Chain and Binomial Broadcast

In the case of the Segmented Chain Broadcast, however, this phenomenon affects all message sizes. Because large messages are split into small segments, such segments suffers from the same transmission delays as the Binomial Broadcast with small messages. Further, due to the Chain structure, a delay in one node is propagated until the end of the chain. Nevertheless, the transmission delay 
for a large message (and by consequence, a large number of segments) does not increases proportionally as it would be expected, but remains constant.

We believe that because these transmission delays are related to the buffering policy from TCP, we believe that the first segments that arrive are delayed by the TCP acknowledge policy, but the successive arrival of the following segments forces the transmission of the remaining segments without any delay.

\subsection{Scatter}

In the case of Scatter, we compare the experimental results from Flat and Binomial Scatters with the predictions from their models. Due to our network characteristics, our experiments shown that a Binomial Scatter can be more efficient than Flat Scatter, a fact that is not usually explored by traditional MPI implementations. As a Binomial Scatter should balance the cost of combined messages and parallel sends, it might occur, as in our experiments, that its performance outweighs the "simplicity" from the Flat Scatter with considerable gains according to the message size and number of nodes, as shown in Figs. 3 (a) and 3 (b). In fact, the Flat Tree model is limited by the time the root needs to send successive messages to different nodes (the gap), while the Binomial Tree Scatter depends mostly on the number of nodes, which defines the number of communication steps through the $\left\lceil\log _{2} P\right\rceil \times L$ factor. These results show that the communication models we developes are accurate enough to identify which implementation is the best adapted to a specific environment and a set of parameters (message size, number of nodes).

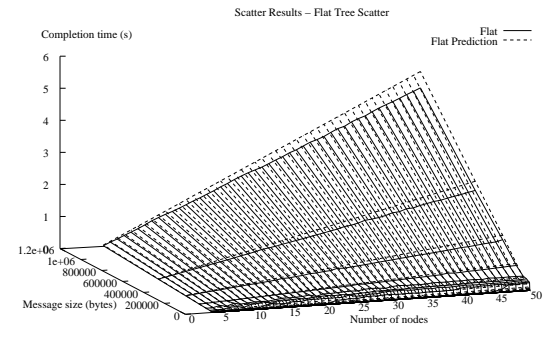

(a) Flat Tree Scatter

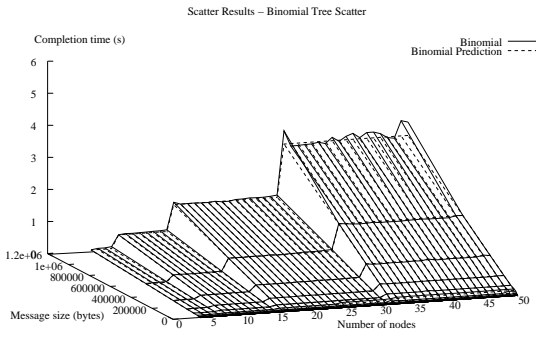

(b) Binomial Tree Scatter

Figure3. Comparison between models and real results

Further, although we can observe some delays related to the TCP acknowledgement policy on Linux when messages are small, specially in the Flat Scatter, these variations are less important than those from the Broadcast, as depicted in Fig. 因.

What called our attention, however, was the performance of the Flat Tree Scatter, that outperformed our predictions, while the Binomial Scatter follows 


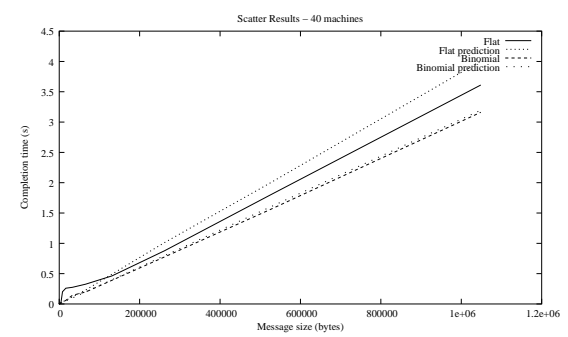

Figure4. Comparison between Flat and Binomial Scatter

the predictions from its model. We think that the multiple transmissions from the Flat Scatter become a "bulk transmission", which forces the communication buffers to transfer the successive messages all together, somehow similarly to the successive sends on the Segmented Chain Broadcast. Hence, we observe that the pLogP parameters measured by the pLogP benchmark tool are not adapted to such situations, as it considers only individual transmissions, mostly adapted to the Binomial Scatter model.

This behaviour seems to indicate a relationship between the number of successive messages sent by a node and the buffer transmission delay, which are not considered in the pLogP performance model. As this seem a very interesting aspect for the design of accurate communication models, we shall closely investigate and formalise this "multi-message" behaviour in a future work.

\section{Conclusions and Future Works}

Existing works that explore the optimisation of heterogeneous networks usually focus only the optimisation of inter-cluster communication. We do not agree with this approach, and we suggest to optimise both inter-cluster and intracluster communication. Hence, in this paper we described how to improve the communication efficiency on homogeneous cluster through the use of well known implementation strategies.

To compare different implementation strategies, we rely on the modelling of communication patterns. Our decision to use communication models allows a fast and accurate performance prediction for the collective communication strategies, giving the possibility to choose the technique that best adapts to each environment. Additionally, because the intra-cluster communication is based on static techniques, the complexity on the generation of optimal trees is restricted only to the inter-cluster communication.

Nonetheless, as our decisions rely on network models, their accuracy needs to be evaluated. Hence, in this paper we presented two examples that compare the predicted performances and the real results. We shown that the selection of the best communication implementation can be made with the help of the communication models. Even if we found some small variations in the predicted data for small messages, these variations were unable to compromise the final 
decision, and we could identify the probable origin from these variations. Hence, one of our future works include a deep investigation on the factors that lead to such variations, and in special the relationship between the number of successive messages and the transmission delay, formalising it and proposing extensions to the pLogP model.

In parallel, we will evaluate the accuracy of our models with other network interconnections, specially Ethernet $1 \mathrm{~Gb}$ and Myrinet, and study how to reflect the presence of multi-processors and multi-networks (division of traffic) in our models. Our research will also include the automatic discovery of the network topology and the construction of optimised inter-cluster trees that work together with efficient intra-cluster communication.

\section{References}

1. Barnett, R., Payne, D., van de Geijn, R., Watts, J.: Broadcasting on meshes with wormhole routing. Journal of Parallel and Distributed Computing, Vol 35, No 2,Academic Press (1996) 111-122

2. Bhat, P., Raharendra, C., Prasanna, V.: Efficient Collective Communication in Distributed Heterogeneous Systems. Journal of Parallel and Distributed Computing, No. 63, Elsevier Science. (2003) 251-263

3. Karonis, N. T., Foster, I., Supinski, B., Gropp, W., Lusk, E., Lacour, S.: A Multilevel Approach to Topology-Aware Collective Operations in Computational Grids. Technical report ANL/MCS-P948-0402, Mathematics and Computer Science Division, Argonne National Laboratory (2002).

4. Kielmann, T., Hofman, R., Bal, H., Plaat, A., Bhoedjang, R.: MagPIe: MPI's Collective Communication Operations for Clustered Wide Area Systems. In: Proceeding Seventh ACM SIGPLAN Symposium on Principles and Practice of Parallel Programming, Atlanta, GA, ACM Press. (1999) 131-140

5. Kielmann, T., Bal, H., Verstoep, K.: Fast Measurement of LogP Parameters for Message Passing Platforms. In: 4th Workshop on Runtime Systems for Parallel Programming (RTSPP), Cancun, Mexico, Springer-Verlag, LNCS Vol. 1800 (2000) 1176-1183. http://www.cs.vu.nl/albatross/

6. Kielman, T., Bal, E., Gorlatch, S., Verstoep, K, Hofman, R.: Network Performanceaware Collective Communication for Clustered Wide Area Systems. Parallel Computing, Vol. 27, No. 11, Elsevier Science. (2001) 1431-1456

7. LAM-MPI Team, LAM/MPI Version 6.5.9, http://www.lam-mpi.org/ (2003)

8. LAM-MPI Team, LAM/MPI Version 7, http://www.lam-mpi.org/ (2004)

9. LAM-MPI Team, Performance Issues with LAM/MPI on Linux 2.2.x, http://www.lam-mpi.org/linux/ (2001)

10. Loncaric, J.: Linux TCP Patches to improve acknowledgement policy. http://research.nianet.org/ josip/LinuxTCP-patches.html (2000)

11. Lowekamp, B.: Discovery and Application of Network Information. PhD Thesis, Carnegie Mellon University. (2000)

12. Thakur, R., Gropp, W.: Improving the Performance of Collective Operations in MPICH. In: Proceedings of the Euro PVM/MPI 2003. Springer-Verlag, LNCS Vol. 2840. (2003) 257-267

13. Vadhiyar, S., Fagg, G., Dongarra, J.: Automatically Tuned Collective Communications. In: Proceedings of Supercomputing 2000, Dallas TX. IEEE Computer Society (2000) 